\title{
Kräuterbücher (5)
}

Einblicke in das Alchemielabor: Zwei bisher unbekannte Alchemistenhandschriften

\author{
Martin Kluge
}

In dieser Reihe um Kräuterbuch-Raritäten aus dem Nachlass des ETH-Professors Carl Hartwich (1851-1917) [1] wurden bisher vor allem botanische Schriften vorgestellt. In den Beständen seiner Bibliothek befanden sich aber auch einige wenige Handschriften, darunter zwei alchemistische aus dem Umfeld paracelsistischer Autoren, die diesmal Gegenstand der Betrachtung werden sollen. Da die Handschriften bisher nicht bearbeitet wurden, sind sie der Forschung bislang verborgen geblieben.

Die erste hier vorgestellte Handschrift mit der Inventarnummer 188 beginnt mit dem vielversprechenden Titel «De Occulta Philosophia, Oder Von der heimlichen Wundergeburt der sieben Planeten und Metalle», endet jedoch überraschend mit den Worten «Herausgegeben und getrukt Hamburg, In Verlegung Samuel Heyls, getrukt mit Sel. Georg Königs Schrifften 1717» (Abb. 1). Bei dieser «Occulta Philosophia» handelt es sich um eines der Schlüsselwerke des Alchemisten Basilius Valentinus. Im Druck ist der Text erstmals 1603 erschienen. Und tatsächlich folgt der Wortlaut der Handschrift exakt der gedruckten Gesamtausgabe des Valentinus von 1717.

\section{Abschriften von Texten des Basilius Valentinus}

Es folgen weitere Traktate von Valentinus in der Reihenfolge der Gesamtausgabe: «Von den natürlichen und übernatürlichen Dingen» oder «Extract aus dem Triumpf-Wagen-Antimonii», danach eine Reihe Supplemente. Dass gedruckte Bücher auch rund 250 Jahre nach Gutenbergs Erfindung des Buchdrucks noch von Hand abgeschrieben wurden, ist keine Seltenheit, sofern die Werke schwer zu erhalten waren. In den hier vorliegenden Abhandlungen beschreibt Valentinus alchemistische Vorstellungen, wonach die Metalle nicht feste, unveränderliche Elemente darstellen, sondern unterschiedliche Zustandsformen ein und derselben Ausgangsmaterie, einer Prima Materia, sind. Demnach lässt sich Materie durch verschiedene Wandlungsstufen so lange von einem

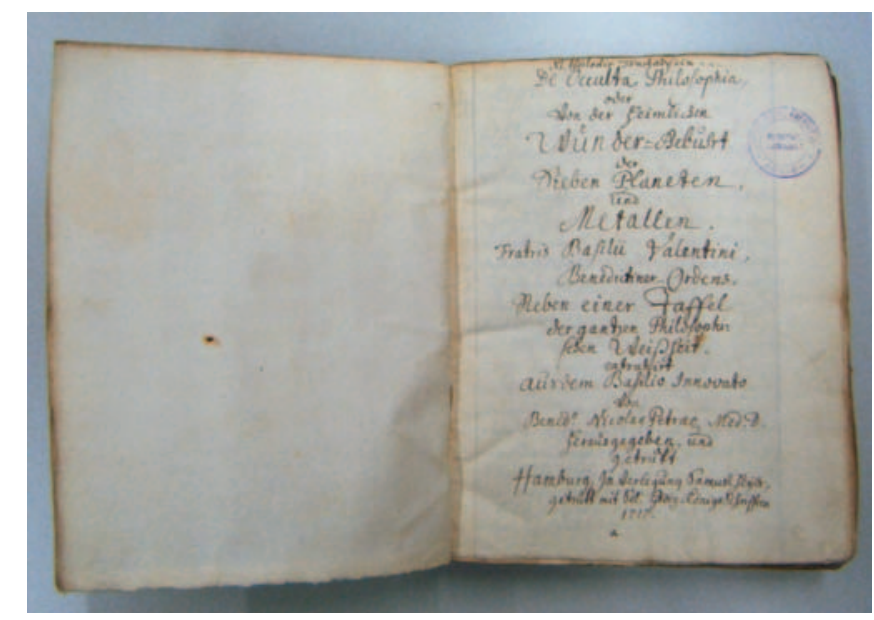

Abb. 1. Titelseite der Handschrift Nr. 188 aus dem Nachlass von Carl Hartwich (Foto: Pharmazie-Historisches Museum Basel).

Metall ins andere umwandeln, bis sich am Ende als höchste Stufe Gold darstellen lässt.

Basilius Valentinus galt einst als ein bekannter Autor, der selbst dem an Alchemie interessierten Johann Wolfgang von Goethe ein Begriff war. Er soll seit 1413 in Erfurt als Benediktinermönch gewirkt haben [2]. Inhaltlich weisen seine Schriften zahlreiche Übereinstimmungen mit den Lehren des Paracelsus auf. Lange Zeit galt er daher als eigentlicher gedanklicher Urheber der paracelsischen Drei-Prinzipien-Lehre von Mercurius, Sulphur und Sal. Die Rechtfertigungsversuche des Paracelsus, in denen er immer wieder lautstark betont, er habe seine Lehren aufgrund eigener Beobachtungen entwickelt, wurden dahingehend gedeutet, dass Paracelsus versucht haben soll, die Autorenschaft des Basilius Valentinus zu leugnen. Neuere Forschungen gehen hingegen von einer umgekehrten Abhängigkeit aus. Angesichts der Tatsache, dass die Schriften die Kenntnis von Zink (erst seit Georgius Agricola im Jahr 1543 bekannt) voraussetzen und dem Autor die Verwendung des Antimons für Druckertypen (nach 1450) bekannt war, muss sich hinter Basilius Valentinus

\section{KARGER}

Fax +497614520714 Information@Karger.com www.karger.com 
wohl ein fiktiver, deutlich jüngerer Autor verbergen. Wahrscheinlich stammen die Texte aus der Feder des ersten Herausgebers dieser Abhandlungen, Johann Thölde (1565-1614). Dieser war selber Alchemist und trat vor allem als Autor des 1603 erschienenen Standardwerks zur Salzgewinnung, der «Haliographia», in Erscheinung. Er veröffentlichte die Valentinus-Schriften zwischen 1599 und 1604 in Leipzig.

\section{Paracelsus: Querdenker und Wegbereiter}

Mit der Herausgabe alchemistischer Schriften und mit paracelsistischem Gedankengut dürfte sich Thölde ein gutes Geschäft versprochen haben: Paracelsus, der zu Lebzeiten stark angefeindet wurde und einsam in Armut starb, erfuhr zur Wende des 17. Jahrhunderts grosse Verehrung. Seine Werke wurden damals in mehreren Städten herausgegeben, und ihm wurden weitere Schriften untergeschoben.

Der eigentliche Name von Paracelsus ${ }^{*} 1493 / 4$ in Einsiedeln, $\uparrow 1541$ in Salzburg) war Theophrastus Bombastus Aureolus Philippus von Hohenheim. Als Neunjähriger zog er mit seinem Vater nach Villach in Kärnten, wo er im Bergbau viel über die Gewinnung und Verarbeitung von Metallen lernte. Über seine weitere Ausbildung und frühe akademische Laufbahn ist wenig bekannt. Er gab an, an mehreren Orten in Deutschland, Frankreich und Italien studiert und in Ferrara promoviert zu haben. Sein unstetes Leben führte ihn als Wundarzt auch nach Salzburg, Strassburg und schliesslich 1526/27 nach Basel. Hier gelang es ihm, das Bein des berühmten Druckers Johannes Froben, das die Ärzte bereits aufgegeben hatten, vor der Amputation zu retten. Protegiert durch Froben wurde Paracelsus schliesslich Stadtarzt von Basel, was ihm auch ermöglichte, an der Universität medizinische Vorlesungen zu halten. Da er mit seinen unkonventionellen Ansichten und seiner streitbaren Natur den Ärger der medizinischen Fakultät auf sich zog, musste er die Stadt nach kurzer Zeit fluchtartig in Richtung Colmar verlassen. Nie mehr gelang es ihm, eine ähnlich feste Anstellung zu erhalten. Sein weiterer Weg führte ihn nach Esslingen, Nürnberg, Leipzig, St. Gallen und schliesslich 1541 nach Salzburg, wo er noch im gleichen Jahr starb.

Schon zu Lebzeiten spalteten sich die Meinungen zu Paracelsus. Die einen sahen in ihm einen begnadeten Wunderheiler. Andere verehrten ihn für seine revolutionären Denkweisen über die alchemistische Gewinnung von Heilmitteln und sein umfangreiches theoretisches Gedankengebäude über die Ursachen und Heilung von Krankheiten. Für seine Gegner war er lediglich ein Scharlatan.

Zwei für die damalige Zeit bahnbrechende Erkenntnisse gehen auf Paracelsus zurück: Seine legendäre Aus- sage «Alle Dinge sind Gift, und nichts ist ohne Gift. Allein die Dosis macht, dass ein Ding kein Gift ist» widersprach der damaligen, auf den vier Elementen aufbauenden Weltanschauung. Neuartig war auch seine Strategie, Heilmittel mittels alchemistischer Verfahren für den menschlichen Körper aufzuschliessen bzw. zu extrahieren. Paracelsus bringt die Gewinnung der Arznei als Aufgabe der Alchemie im «Buch Paragranum» (1530) auf eine einfache Formel: «denn die natur ist so subtil / unnd so scharpff inn ihren dingen / daß sie ohn grosse Kunst nicht will gebrauchet werden / Dann sie gibt nichts ann den tag, daß sein statt vollendet sey (= dass in sich selbst vollendet wäre)/ sonder der mensch müß es vollenden / Diese vollendung heisset Alchimia» [3].

\section{Eine weitere bisher unbekannte Alchemistenhandschrift}

Dieses alchemistische Gedankengut und eine Zusammenstellung paracelsistischer Texte finden sich in einer weiteren, bisher unbekannten Handschrift aus der Hartwich-Bibliothek. In der Inventarliste wird sie als Nr. 90 geführt und mit «Von Hand geschrieben. 15. Januar 1608. Manuskript. Historie. Anzahl 1» katalogisiert. Ohne Titelseite oder Inhaltsangabe erschliesst sich der ungewöhnliche Inhalt des Manuskripts erst bei intensiverer Auseinandersetzung mit der zum Teil schwer entzifferbaren Handschrift, über deren Herkunft, Autoren, Schreiber oder Vorbesitzer jedoch keine Angaben zu finden sind. Das durchgehend einheitliche Papier aus einer Augsburger Papiermühle verrät, dass die Texte sukzessive in ein leeres, bereits gebundenes, 144 Seiten umfassendes Journalbuch von $21 \times 31 \mathrm{~cm}$ eingetragen wurden, wie sie von der Mitte des 16. Jahrhunderts an bis in die 1630erJahre im ganzen süddeutschen Raum erworben werden konnten.

Der Inhalt der Handschrift besteht im Wesentlichen aus zwei grösseren Blöcken: einem Journalbuch aus einem alchemistischen Labor und einer Zusammenfassung einiger wichtiger Paracelsus-Schriften.

\section{Arbeitsrapporte aus der Alchemistenküche}

Die Handschrift beginnt mit «Anno Domini Nostri Iesu Christi, 1608, 15. Januar» (Abb. 2) und verrät bereits in der nächsten Zeile, in alchemistischen Zeichen verschlüsselt, worum es geht: «(Wasser) ad (Gold)», Wasser zu Gold machen! Über 3 Seiten folgt ein ausführliches Protokoll über einen Versuch, aus Zinn und Quecksilber ein Goldwasser herzustellen. Dieser Eintrag ist der erste einer sich über 120 Seiten erstreckenden Abfolge von jeweils mit Datum versehenen Aufzeichnungen. Auf alche- 


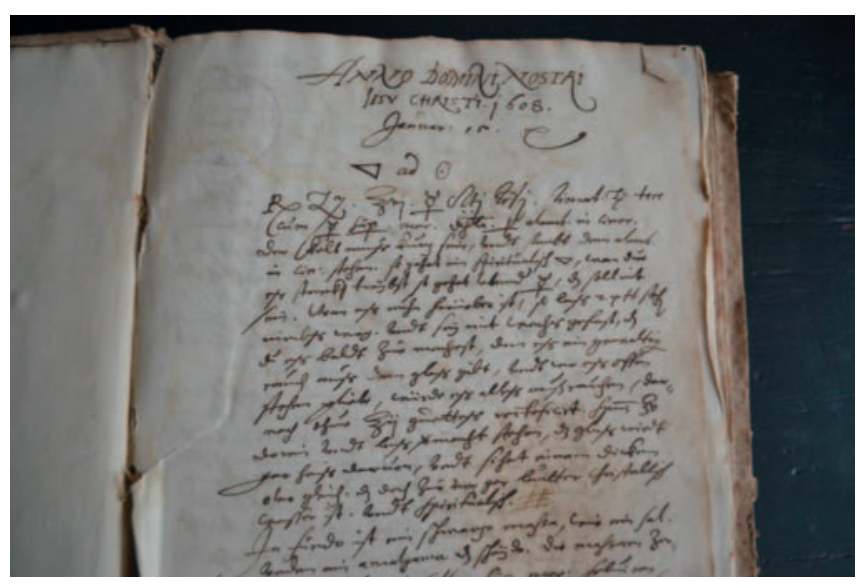

Abb. 2. Anfang des ersten Abschnitts der Handschrift Nr. 90 (Foto: Pharmazie-Historisches Museum Basel).

mistische Weise sollen hier die sieben Metalle (Blei, Eisen, Zinn, Kupfer, Quecksilber, Silber und Gold), aber auch Schwefel oder Salpeter in der anfangs beschriebenen Art «transmutiert» werden, d.h., sie selbst oder ihr «Geist» sollen in Arzneien angereichert oder zu Gold verwandelt werden. Als letztes Datum wurde der 9. Februar 1616 vermerkt.

Die Einträge lassen die Mühen der Arbeiten im Labor erahnen. Auf den ersten Seiten - notiert am 23. Januar 1608 beim Versuch, ein Quecksilber-Salz oder Beljamus Mercury zu gewinnen, welches «wie ein Wunder» sei endet der unbekannte Autor mit den Worten «kostet mich beydes vill glesser vndt mühe biss ich das pordzion finde» (= Kostete mich beides viel: Gläser und Mühe, bis ich die richtige Proportion gefunden habe). Auch seine Versuche vom 10. April 1609, ein Calcium-Gold aus Quecksilber zu bereiten, scheiterten. Resigniert beendet er den Eintrag «hadt dem Gold nichts abgwonnen, wie ich meindt». Auch sein nächster Versuch, mit Kupfersalzen oder einer Zinn-Lösung in der Goldbereitung weiterzukommen, scheiterte: «Nichts, auf dissen wegen das Gold zu destruieren, oder mit nuz zuo solvieren». Erfolgreicher scheinen hingegen seine Versuche für «Ein köstllich Wasser zuo vielen dingen», ebenfalls aus dem Jahr 1609. Der Bericht endet mit den Worten «Das (Wasser) extrahiert Goldt Erzt (= Golderz), undt kann zu vielen dingen gebraucht werden». Ein weiteres von ihm erfolgreich gefundenes Oleum Antimonium sei «Jn Chirugia dienstlig», also in der Chirurgie einsetzbar.

Am 23. Januar 1608 stehen Versuche um Eisen-Extrakte auf dem Programm: zu Extractum Martis (Eisenextrakt) oder Crocus Martis, einem unter Schwefelzugabe entstandenes orangerot leuchtendes bis safranfarbenes Pigment, notierte er die Anmerkung «Alles was ex (Eisen) gemacht wirdt Jst schedlig der leber, dinlich wiederum Jhr Kranckheiten».

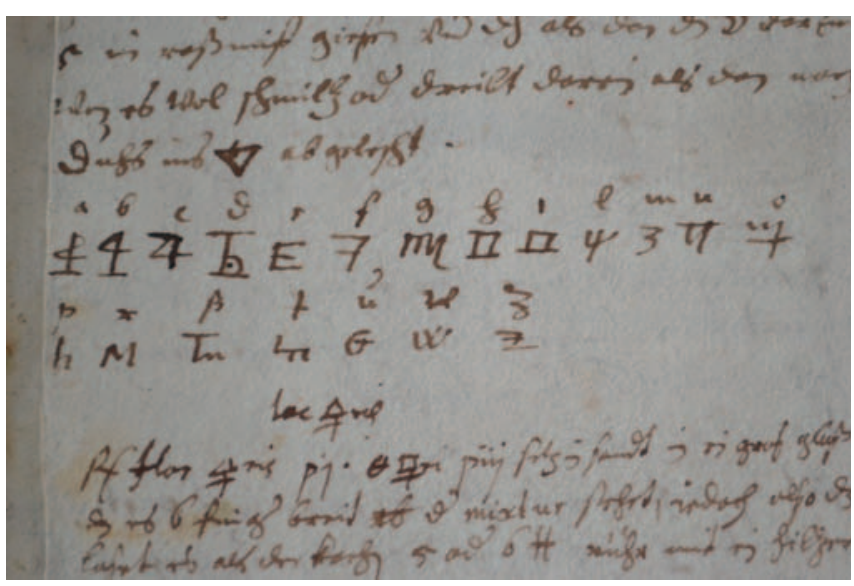

Abb. 3. Anleitung einer Geheimschrift aus alchemistischen Zeichen in der Handschrift Nr. 90 (Foto: Pharmazie-Historisches Museum Basel).

Ein anderer Schreibender mit deutlich feinerer Handschrift ergänzt das Journalbuch um 3 Doppelseiten mit weiteren alchemistischen Rezepturen. Dieser Schreiber empfiehlt auch eine Geheimschrift aus alchemistischen Zeichen (Abb. 3). Nach einer Leerseite beginnt schliesslich der zweite wichtige Abschnitt der Handschrift mit einer neuen Seitenzählung.

\section{Eine nie gedruckte Paracelsus-Handschrift}

Wieder beginnt der Abschnitt mit einer vermeintlichen Titelseite: «Succus Paracelsiacus. die matthai divi Apostoli et evangelista Anno 1626 (= Matthäustag, der 21. September 1626). In Nomine Sacro Santa et Individuae Trinitatis Amen» (Abb. 4). Der lateinische Name Succus bedeutet «Saft» und spielt auf eine traditionelle Arzneiform, den eingedickten, konzentrierten Pflanzensaft, an. Paracelsiacus bedeutet die Lehre des Paracelsus betreffend. Es geht also um eine Zusammenfassung der Lehren des Paracelsus. Der Text ist aber nie im Druck erschienen: Weder ist ein Druck unter dem Titel «Succus Paracelsiacus» bekannt, noch erschien eine Paracelsus-Ausgabe im Jahr 1626.

Der Inhalt des ersten dieser «eingedickten» ParacelsusTraktate erschliesst sich aus den zwei Wörtern der gegenüberliegenden, sonst leeren Seite: PARACELSUS. PARAMIRUM. Im Buch «Volumen Paramirum», einem wichtigen Frühwerk des Paracelsus [4, 5], werden fünf mögliche Ursachen für Krankheiten, die fünf Entia beschrieben: Ens Astrale, unter den Einflüssen der Planeten und der umgebenden Umwelt; das Ens Veneni, für Krankheiten durch Gifte und aufgrund falscher Ernährung; Ens Naturale, wonach Krankheiten durch die eigene Konstitution oder genetische Disposition verursacht werden; Ens Spirituale, durch Magie oder böse geistige Kräfte; und letztlich Ens Die für Krankheiten, die durch göttlichen Willen oder Schicksal entstehen. 


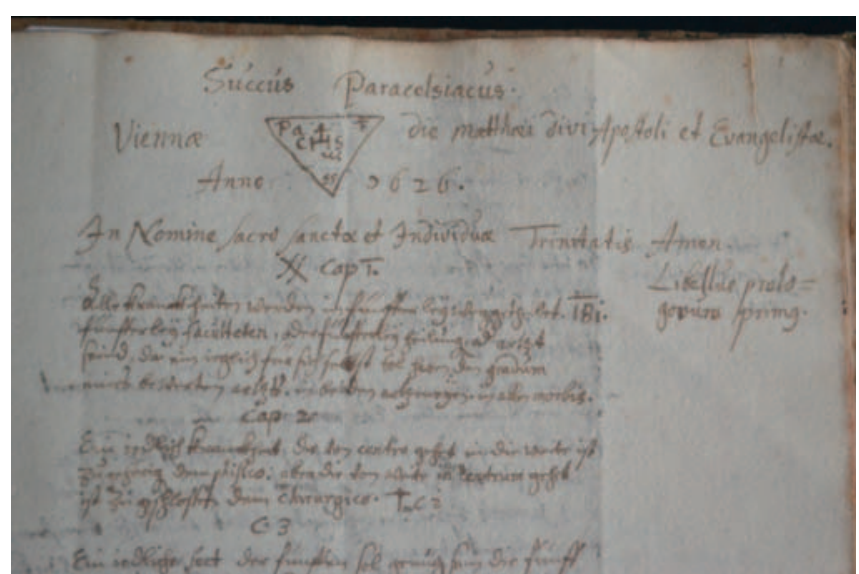

Abb. 4. Anfang des Abschnitts mit verkürzten Paracelsus-Schriften (Foto: Pharmazie-Historisches Museum Basel)

Der Text folgt aber nicht einer der Druckfassungen des 16. Jahrhunderts, sondern gibt ihn - Abschnitt für Abschnitt neu formuliert und auf rund die Hälfte gekürzt - in konzentrierter Form wieder. Das Gleiche gilt auch für weitere, in der Handschrift nachfolgenden ParacelsusTraktate, wie «De origine morborum» (1565 in Köln gedruckt), «De matrice» (ebenfalls 1565 in Köln gedruckt), «De generatione hominis» (1577 in Strassburg gedruckt) sowie «Fragmenta Medica» (1589 in Basel erschienen). Auch diese folgen in einer verkürzten Umschrift. Die um- ständliche Ausdrucksweise von Paracelsus mit seinen ständigen Wiederholungen war auch für Ohren des 17. Jahrhunderts nur schwer verständlich. Daher finden sich Zusammenfassungen oder Neuformulierungen der Ideen des Paracelsus bereits in Handschriften des späten 16. und frühen 17. Jahrhunderts (z.B. Ms. Cambridge (Massachusetts), Harvard College Library, MS. Lat. 221 aus Deutschland, 17. Jh.; Prag, National- und Universitätsbibliothek, MS. VI Fg 1 (Oktav), 16./17. Jh.; Strassburg, Bibliothèque nationale et universitaire, Ms. 2277, aus Basel 16. Jh.) [6]. Ausgerechnet Basel, aus dem Paracelsus einst mit Schimpf und Schande verjagt wurde, sollte nun Ausgangspunkt des neu erwachenden Interesses an seinen Lehren werden. Es war der Basler Medizinprofessor Theodor Zwinger (1533-1588), der sich als Universitätsdozent erstmals wohlwollend in seinen Vorlesungen mit den Thesen Paracelsus' auseinandersetzte. Zwinger war der Neffe von Johannes Oporin, dem einstigen Famulus von Paracelsus in Basel, und kannte die Geschichten um Paracelsus wohl aus erster Hand. In seiner «Physiologie Medica» versuchte er die Thesen von Paracelsus mit eigenen Worten auf den Punkt zu bringen. Um Theodor und seinen Sohn Jakob Zwinger bildete sich in der Folge ein Kreis von Alchemisten und Paracelsisten. Erst postum, durch Zwingers Sohn herausgegeben, erschienen die neuformulierten Thesen um 1610 - nun klarer und schlüssiger, als es Paracelsus je vermochte [7].

\section{Literatur}

1 Brauckmann B: Kräuterbücher (1): Sammelleidenschaft und Forschungseifer: Zwei Gelehrte aus verschiedenen Jahrhunderten auf den Spuren von Opium und Kaffee. Schweiz Z Ganzheitsmed 2014;26:105-107.

2 Deutsche Biographie: Basilius Valentinus. www.deutsche-biographie.de/sfz2188.html.

3 von Bodenstein A: Das Buch Paragranum Aureoli Theophrasti Paracelsi: Darinn die vier Columnae, als das ist / Philosophia, Astronomia, Alchimia unnd Virtus, auff wel- che Theophrast Medicinn fundirt ist / tractirt werden. Zitiert nach Paracelsus. Frankfurt, Egenolff, 1565.

4 Strunz F: Theophrastus Paracelsus: Volumen Paramirum und Opus Paramirum. Jena, 1904.

5 Strebel J: Theophrastus von Hohenheim genannt Paracelsus. Sämtliche Werke in zeitgemässer Kürzung Band V: Propädeutische Schriften: Paragranum, Volumen und Opus Paramirum mit Appendix (Schweizer bzw. St. Galler Band). St. Gallen, 1947.
6 Weimann K-H: Neu entdeckte ParacelsusHandschriften. Sudhoffs Arch Gesch Med Naturwiss 1957;41:154-159.

7 Gantenbein UL: Die Geschichte der Schweizerischen Paracelsus-Gesellschaft. Salzburger Beitr Paracelsusforsch 1999;33:84-109. 